\title{
Perioperative beta-blockade: might the baby go out with the bath water?
}

\author{
Authors: Ravin Mistry ${ }^{A}$ and David Walker ${ }^{B}$
}

\begin{abstract}
Perioperative $\beta$-blocker therapy has been advocated to reduce cardiac mortality and morbidity in high-risk cardiac patients undergoing non-cardiac surgery. Core data that supported this intervention and informed international societal guidelines has recently been withdrawn. A subsequent meta-analysis of the remaining data reporting excess mortality has re-opened the debate about the utility of $\beta$-blocker therapy in the perioperative period. Criticism of remaining trial designs and new insights into the protective mechanisms of $\beta$-blocker therapy in critical illness raise important questions that should now be addressed by a further robust, high-quality randomised control trial.
\end{abstract}

KEYWORDS: $\beta$-blockers, perioperative, non-cardiac surgery.

\section{Introduction}

The systemic inflammatory response associated with major surgery is well characterised and impacts on outcome in the perioperative period. This is particularly apparent in those patients who suffer significant systemic co-morbidity who present for major surgery. This group, termed the 'high-risk surgical population', are relatively small in absolute numbers but account for the majority of the morbidity and mortality in the perioperative period. ${ }^{1}$ Cardiopulmonary insufficiency is considered an important determinant of outcome, and much attention in recent times has focused on cardiac protection and optimisation in the perioperative period.

The Multicenter Study of Perioperative Ischemia of 1996 (McSPI, 1996) ${ }^{2}$ and the later Dutch Echocardiographic Cardiac Risk Evaluation Applying Stress Echocardiography Study Group I (DECREASE I, 1999) ${ }^{3}$ were the first trials to demonstrate the value of $\beta$-blockers in the perioperative period. Both trials demonstrated a reduced cardiovascular morbidity and mortality in populations of high-risk patients undergoing non-cardiac surgery.

The McSPI trial investigated the intervention of atenolol in non-cardiac surgical patients with known coronary artery disease; it randomised them to placebo or intravenous atenolol,

Authors: A perioperative medicine fellow/ST6 anaesthetics registrar, Anaesthetics Department, University College Hospital NHS Foundation Trust, London, UK; ${ }^{B}$ consultant in anaesthesia and intensive care, Anaesthetics Department, University College Hospital NHS Foundation Trust, London, UK

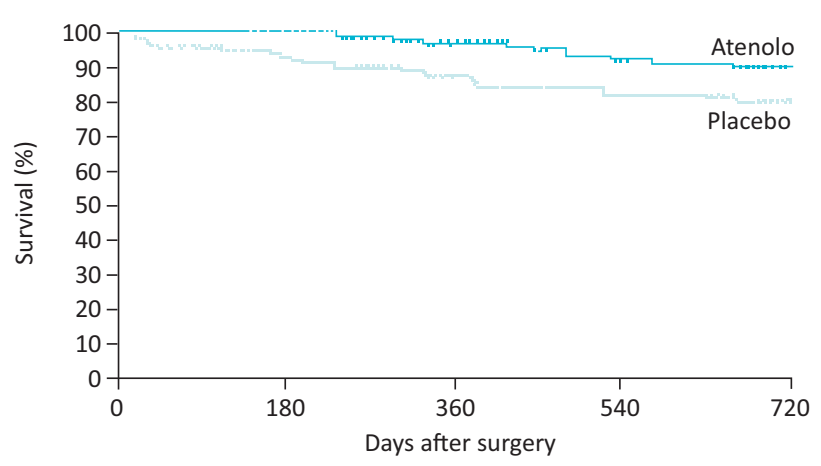

Fig 1. Overall survival in the two years after non-cardiac surgery among 192 patients in the atenolol and placebo groups who survived to hospital discharge. ${ }^{2}$

pre-induction of anaesthesia. The intervention, which was continued for 7 days postoperatively, was associated with a significant reduction in all-cause mortality at 2 years and a reduction in the incidence of non-fatal cardiac complications (Fig 1). The study attracted widespread criticism because patients who had already been prescribed long-term $\beta$-blocker therapy were included in the trial and, if randomised to the placebo arm, had their therapy abruptly stopped. In addition, only those patients who survived to hospital discharge were included in the final analysis. Subsequent intention-to-treat analysis of the data demonstrated an insignificant difference in mortality rate at 2 years between the control and atenolol groups.

The DECREASE I study prospectively assessed the effect of perioperative bisoprolol, as compared with placebo, in patients undergoing major vascular surgery. Therapy was commenced, on average, one week preoperatively and continued for 30 days postoperatively. Importantly, the study protocol included a dose titration that was based on targeting a pre-defined heart rate range. The study demonstrated a clear reduction in mortality from cardiac causes and non-fatal myocardial infarction within 30 days of surgery (34\% in the placebo group vs. $3.4 \%$ in the intervention group). A clear benefit was demonstrated in the intervention group, and thus the trial was stopped early after enrolling only 112 patients. Once again, however, the trial design attracted several criticisms, including the failure to use a double-blinded methodology.

Encouraged by the results of DECREASE I, the same group of investigators undertook further trials. This family of studies 


\begin{tabular}{|c|c|c|}
\hline Trial & Risk category & Conclusion \\
\hline DECREASE I & High & $\begin{array}{l}\text { In high-risk patients undergoing non-cardiac surgery, perioperative } \beta \text {-blockade with bisoprolol } \\
\text { significantly reduces cardiac death and MI in the short- and long-term }\end{array}$ \\
\hline DECREASE II & Low, intermediate, high & $\begin{array}{l}\text { Patients identified as intermediate risk on the basis of a simple clinical assessment do not } \\
\text { need pre-operative echocardiographic cardiac stress testing, provided that they receive } \\
\text { bisoprolol to maintain resting heart rate at } 60-65 \text { bpm }\end{array}$ \\
\hline DECREASE III & High & $\begin{array}{l}\text { In high-risk patients undergoing major vascular surgery, fluvastatin XL significantly reduces } \\
\text { myocardial ischaemia and the combined endpoint of cardiovascular death and MI }\end{array}$ \\
\hline DECREASE IV & Intermediate & $\begin{array}{l}\text { In intermediate-risk patients, bisoprolol significantly reduces cardiac death and MI, with a } \\
\text { non-significant trend towards a beneficial effect of fluvastatin XL }\end{array}$ \\
\hline DECREASE V & High & $\begin{array}{l}\text { In high-risk patients with extensive stress-induced ischaemia, coronary revascularisation } \\
\text { (added to tight heart rate control with bisoprolol) does not produce any additional reduction } \\
\text { in death and MI and delays surgery. }\end{array}$ \\
\hline
\end{tabular}

continued to demonstrate the benefits of both bisoprolol and statins in intermediate- and high-risk non-cardiac surgery patients (Table 1).

Despite the criticisms of these trials, the ground swell of support for perioperative $\beta$-blocker therapy led to the publication of the first formal guidance on perioperative cardiovascular evaluation in non-cardiac surgery from the American College of Cardiology and the American Heart Association (ACC/AHA). ${ }^{5}$ Based predominantly on the findings of the McSPI and DECREASE trials, these guidelines cited class 1 recommendation for $\beta$-blocker therapy in patients with previous $\beta$-blocker use for hypertension, angina or arrhythmia, or for those deemed of high cardiac risk on preoperative cardiac testing.

By contrast, the first negative trials questioning the role of $\beta$-blockers came in 2006 with the Diabetic Postoperative Mortality and Morbidity (DIPOM) ${ }^{6}$ and Metoprolol after Vascular Surgery (MaVS) studies. ${ }^{7}$ DIPOM randomised diabetic patients to placebo or sustained-release metoprolol, to be commenced 24 hours prior to surgery and continuing up to 8 days postoperatively. MaVS, a double-blinded randomised controlled trial, randomised patients to placebo or metoprolol, to begin 2 hours preoperatively and continuing for up to 5 days after surgery. Both trials titrated $\beta$-blocker therapy to heart rate and systolic blood pressure. The sample size of these combined trials (1,417 patients) surpassed that of the McSPI and DECREASE combined sample size by a factor of four. Both studies failed to demonstrate a significant reduction in cardiovascular mortality in response to metoprolol, but found an increased incidence of hypotension and bradycardia requiring treatment in the metoprolol group.

In 2008, the Canadian-based Perioperative Ischemic Evaluation (POISE) ${ }^{8}$ study was born out of an increasing scepticism and conflicting evidence regarding the benefits of perioperative $\beta$-blockers in non-cardiac surgery. This study was the largest of its kind and designed to be powered adequately to detect a $25 \%$ relative risk reduction in cardiovascular death or cardiac complications. The study enrolled 8,351 patients with or at risk of atherosclerotic heart disease and randomised them to placebo or extended release (ER) metoprolol immediately before 'high risk', non-cardiac surgery and continuing for 30 days postoperatively. Outcomes measured at 30 days showed a statistically significant reduction in myocardial infarction rates (4.2\% metoprolol vs. 5.7\% placebo), a reduced need for coronary revascularisation and a reduction in the number of patients developing atrial fibrillation. More importantly, however, there was a significant increase in overall mortality (3.1\% metoprolol vs. $2.3 \%$ placebo) and stroke ( $1 \%$ metoprolol vs. $0.5 \%$ placebo), as well as increased episodes of clinically significant hypotension and bradycardia. The authors concluded that for every 1,000 patients undergoing non-cardiac surgery, metoprolol would prevent 15 myocardial infarctions, 3 coronary revascularisations and 7 patients' developing clinically significant atrial fibrillation. Adversely, metoprolol would cause an excess of 8 patients to die and 5 to suffer a stroke for every 1,000 patients treated.

In spite of what appeared to be a well-conducted trial, the results were overshadowed by criticism of the dosing methodology employed. Trial protocols dictated that patients could potentially be prescribed a total of $400 \mathrm{mg}$ of metoprolol ER on the day of surgery, a dose and formulation considered by many to be excessive for a ' $\beta$-blocker-naïve population'. The timing of the introduction of $\beta$-blocker therapy was also questioned. In line with other studies, the intervention commenced only 2-4 hours preoperatively, allowing little time for physiological adaption before the commencement of anaesthesia and surgery.

In 2009, the ACC/AHA downgraded their recommendations for perioperative $\beta$-blockade, stating 'In light of the POISE results, routine administration of perioperative beta blockers, particularly in higher fixed-dose regimens begun on the day of surgery, cannot be advocated.'

Largely on the basis of the DECREASE family of studies and in contrast to the ACC/AHA downgrade, the European Society of Cardiology (ESC), proposed class 1 recommendations for the commencement of $\beta$-blockers in patients with significant risk factors for atherosclerotic disease who are undergoing high- and intermediate-risk surgery. ${ }^{10}$

In 2011, concerns were raised regarding the scientific credibility of data from the DECREASE VI trial and subsequent adjudication concluded that the DECREASE family of trials were conducted in a manner that was 'negligent and scientifically incorrect.' ${ }^{11,12}$ These discredited data were no longer valid and were considered unreliable evidence to 
support the perioperative use of $\beta$-blocker therapy. The earlier DECREASE I study was not investigated as it had been carried out beyond the 10-year deadline for investigation.

In 2013, the remaining studies on the preoperative use of $\beta$-blockers, excluding the discredited DECREASE studies, were subjected to a new meta-analysis. ${ }^{13}$ The primary outcome examined in this analysis remained all-cause mortality at 30 days or at hospital discharge. On the basis of this new meta-analysis, the initiation of $\beta$-blockers preoperatively was associated with a $27 \%$ risk increase in 30 -day all cause mortality (95\% CI 1.01-1.60, p=0.04) and with reduced non-fatal myocardial infarction (relative risk reduction 0.73 , $\mathrm{p}=0.001$ ), but with increased risk of a cerebrovascular accident (CVA) (RR 1.73, p=0.05) and hypotensive episodes (RR 1.51, $\mathrm{p}<0.00001)$. The authors extrapolated that if current ESC guidelines were to be strictly adhered to in the UK, where almost 2.5 million intermediate- or high-risk procedures are carried out annually, there could be 10,000 potentially avoidable deaths in high-risk surgical patients in the UK.

Despite the discreditation of the DECREASE trial data in 2011, and this latest meta-analysis showing the potential harm in using perioperative $\beta$-blockers, the ESC have chosen to allow their 2009 class recommendations to remain in place until now. ${ }^{14}$ The softening of their recommendations for initiation of perioperative $\beta$-blockade from class I to class IIb, with an insistence that this recommendation is underpinned by evidence that excludes the DECREASE I trial data, has been strongly contested recently. ${ }^{15}$

\section{Is there any role for perioperative $\beta$-blocker therapy?}

Perhaps not surprisingly, the use of $\beta$-blockers in high-risk surgery has declined in recent times, ${ }^{16}$ which may reflect confusion within the perioperative medicine community regarding the evidence base on which to proceed.

Recently, evidence from both the perioperative and critical care community again shines a light on $\beta$-blocker therapy, suggesting that it may have an important role to play in improving outcomes.

In 2013, London et al ${ }^{17}$ performed a retrospective, propensitymatched analysis of 136,745 patients from the American Veterans Affairs Surgical Quality Improvement (VASQIP) database who were treated over a 6 -year period. This analysis demonstrated that among propensity-matched individuals, exposure to $\beta$-blockers on the day of or following major non-cardiac surgery was associated with lower rates of 30-day all-cause mortality, non-fatal Q-wave myocardial infarction and cardiac arrest. This finding was more likely in patients with two or more Revised Cardiac Index risk factors (ie in those with a high-risk surgery, cerebrovascular disease, ischaemic heart disease, congestive heart failure, diabetes mellitus, or renal insufficiency). Withdrawal of long-term $\beta$-blocker therapy in the perioperative period was associated with a higher mortality, similar to the findings seen in DECREASE I for those patients using long-term $\beta$-blocker therapy who were randomised to the placebo arm of the trial. Interestingly, and contrary to the findings of previous studies, no benefit was seen in those patients undergoing vascular surgery - a group considered to be most at risk of significant perioperative cardiac events and who might benefit most from intervention. In contrast to POISE, this analysis was unable to demonstrate a higher risk of CVA with the exposure to $\beta$-blockers, which may reflect the dosing strategy employed in the POISE trial.

Observational data from both those with severe brain injury and critically ill trauma patients has demonstrated that premorbid $\beta$-blocker use is associated with decreased hospital mortality. ${ }^{18,19}$ In critical illness, $\beta$-blockers are considered by many to have potential as an attractive intervention. The evidence is largely experimental and evolving, but there is renewed interest in their role in the treatment of sepsis, where overwhelming sympathetically mediated catecholamine excess may lead to direct myocardial damage. In a population of patients with septic shock, ${ }^{20}$ esmolol intervention was associated with an increase in 28 -day survival when used in titration to target a heart rate of $80-94$ beats per minute. The treatment group had an increased stroke volume and a reduction in the dose of exogenous vasopressor, and maintained blood pressure without the need for additional inotropic support. The authors postulated that improved coronary filling time and intracellular calcium handling contributed to reduced cardiac work and myocyte toxicity, providing potential mechanisms for improved outcome. In a re-examination of the evidence for $\beta$-blocker use in sepsis, Novotny et al ${ }^{21}$ highlight the potential noncardiac effects of $\beta$-blockers, including their effects on cytokine production, the inflammatory cascade and improved cellular metabolism and efficiency. Such findings raise interesting questions about the utility of $\beta$-blockade in other inflammatory illnesses, including recovery from surgery. If the presumed physiological responses to therapy have a 'protective' role to play in perioperative outcomes, it is conceivable that starting a 'onesize fits all' therapy at time of surgery, without a period of host modulation or adaption, might not be optimal.

\section{Conclusions}

The role of $\beta$-blockers in reducing the risk of major adverse cardiac events in high-risk patients undergoing non-cardiac surgery is unclear and fundamental questions remain unanswered. The timing and dose of intervention, the optimal selection of drugs based on pharmacodynamics, the appropriate outcomes to be measured and, not least, the population most likely to benefit from treatment remain important factors that can only be answered by a new large well-conducted randomised control trial. The use of $\beta$-blockers in critically ill patients has provided exciting observations, which may challenge our understanding of pathophysiology and the mechanisms by which therapy might alter outcomes. Ultimately, a position of professional equipoise over the use of $\beta$-blockers in the perioperative period should drive a process of further investigation through more appropriate trial design.

\section{Competing interests}

No competing interests.

\section{References}

1 Pearse RM, Harrison DA, James $\mathrm{P}$ et al. Identification and characterisation of the high-risk surgical population in the United Kingdom. Crit Care 2006;10:R81.

2 Mangano DT, Layug EL, Wallace A, Tateo I. Effect of atenolol on mortality and cardiovascular morbidity after noncardiac surgery. Multicenter Study of Perioperative Ischemia Research Group. N Engl J Med 1996;335:1713-20. 
3 Poldermans D, Boersma E, Bax JJ et al. The effect of bisoprolol on perioperative mortality and myocardial infarction in high-risk patients undergoing vascular surgery. Dutch Echocardiographic Cardiac Risk Evaluation Applying Stress Echocardiography Study Group. N Engl J Med 1999;341:1789-94.

4 Poldermans D, Schouten O, Bax J, Winkel TA. Reducing cardiac risk in non-cardiac surgery: evidence from the DECREASE studies. Eur Heart J 2009;11(Suppl A):A9-A14.

5 Eagle KA, Berger PB, Calkins $\mathrm{H}$ et al. ACC/AHA guideline update for perioperative cardiovascular evaluation for noncardiac surgery - executive summary. A report of the American College of Cardiology/American Heart Association Task Force on Practice Guidelines (Committee to Update the 1996 Guidelines on Perioperative Cardiovascular Evaluation for Noncardiac Surgery). Anesth Analg 2002;94:1052-64.

6 Juul AB, Wetterslev J, Gluud C et al. Effect of perioperative beta blockade in patients with diabetes undergoing major non-cardiac surgery: randomised placebo controlled, blinded multicentre trial. Br Med J 2006;332:1482.

7 Yang H, Raymer K, Butler R, Parlow J, Roberts R. The effects of perioperative beta-blockade: results of the Metoprolol after Vascular Surgery (MaVS) study, a randomized controlled trial. Am Heart $J$ 2006;152:983-90.

8 Devereaux PJ, Yang H, Yusuf S et al. Effects of extended-release metoprolol succinate in patients undergoing non-cardiac surgery (POISE trial): a randomised controlled trial. Lancet 2008; 371:1839-47.

9 Fleisher LA, Beckman JA, Brown KA et al. 2009 ACCF/AHA focused update on perioperative beta blockade incorporated into the ACC/AHA 2007 guidelines on perioperative cardiovascular evaluation and care for noncardiac surgery: a report of the American college of cardiology foundation/American heart association task force on practice guidelines. Circulation 2009;120:e169-e276.

10 Poldermans D, Bax JJ, Boersma E et al. Guidelines for pre-operative cardiac risk assessment and perioperative cardiac management in non-cardiac surgery: the task force for preoperative cardiac risk assessment and perioperative cardiac management in non-cardiac surgery of the European Society of Cardiology (ESC) and endorsed by the European Society of Anaesthesiology (ESA). Eur J Anaesthesiol 2010;27:92-137.
11 Erasmus Medical Centre, 2011. Investigation into possible violation of scientific integrity. Available online at www.erasmusmc.nl/5663/135857/ 3664573/3397899/report_summary_investigation_integrity [Accessed 5 August 2014].

12 Erasmus Medical Centre, 2012. Report on the 2012 follow-up investigation of possible breaches of academic integrity. Available online at http://cardiobrief.files.wordpress.com/2012/10/integrityreport-2012-10-english-translation.pdf [Accessed 5 August 2014].

13 Bouri S, Shun-Shin MJ, Cole GD et al. Meta-analysis of secure randomised controlled trials of $\beta$-blockade to prevent perioperative death in non-cardiac surgery. Heart 2014;100:456-64.

14 Joint Task Force on Non-Cardiac Surgery Cardiovascular Assessment and Management of the European Society of Cardiology (ESC) and the European Society of Anaesthesiology (ESA). 2014 ESC/ESA guidelines on non-cardiac surgery: cardiovascular assessment and management. Eur Heart J 2014;35:2383-431.

15 Cole G, Francis D. Perioperative $\beta$ blockade: guidelines do not reflect the problems with the evidence from the DECREASE trials. BMJ 2014;349:g5210.

16 Wijeysundera DN, Mamdani M, Laupacis A et al. Clinical evidence, practice guidelines, and $\beta$-blocker utilization before major noncardiac surgery. Circ Cardiovasc Qual Outcomes 2012;5:558-65.

17 London MJ, Hur K, Schwartz GG, Henderson WG. Association of perioperative beta-blockade with mortality and cardiovascular morbidity following major noncardiac surgery. J Am Med Assoc 2013;309:1704-13.

18 Mohseni S, Talving P, Wallin G et al. Preinjury A-blockade is protective in isolated severe traumatic brain injury. J Trauma Acute Care Surg 2014;76:804-8.

19 Bukur M, Lustenberger T, Cotton B et al. Beta-blocker exposure in the absence of significant head injuries is associated with reduced mortality in critically ill patients. Am J Surg 2012;204:697-703.

20 Morelli A, Ertmer C, Westphal M et al. Effect of heart rate control with esmolol on hemodynamic and clinical outcomes in patients with septic shock: a randomized clinical trial. JAMA 2013;310:1683-91.

21 Novotny NM, Lahm T, Markel TA et al. Beta-blockers in sepsis: re-examining the evidence. Shock 2009;31:113-9.

Address for correspondence: Dr R Mistry, Flat 15 High Trees Mansions, 28 Crescent Wood Rd, London SE26 6RU, UK.

Email: ravin.mistry@nhs.net 